\title{
Recent advances in the pathogenesis and treatment of osteoporosis
}

\author{
Authors: Elizabeth M Curtis, ${ }^{\mathrm{A}}$ Rebecca J Moon, ${ }^{\mathrm{B}}$ Elaine M Dennison, ${ }^{\mathrm{C}}$ Nicholas C Harvey ${ }^{\mathrm{D}}$ and Cyrus Cooper ${ }^{\mathrm{E}}$
}

\begin{abstract}
Over recent decades, the perception of osteoporosis has changed from that of an inevitable consequence of ageing, to that of a well characterised and treatable chronic non-communicable disease, with major impacts on individuals, healthcare systems and societies. Characterisation of its pathophysiology from the hierarchical structure of bone and the role of its cell population, development of effective strategies for the identification of those most appropriate for treatment, and an increasing armamentarium of efficacious pharmacological therapies, have underpinned this evolution. Despite this marked progress, individuals who experience a fragility fracture remain under-treated in many areas of the world, and there is substantial need for investment both in secondary and primary prevention globally. In this brief article, we give an overview of the pathogenesis of osteoporosis, and summarise current and future approaches to its assessment and treatment.
\end{abstract}

KEYWORDS: osteoporosis, epidemiology, pathophysiology, treatment, risk assessment, $\mathrm{dxa}$

\section{Introduction}

Osteoporosis is characterised by deterioration of bone mass and microarchitecture, resulting in increased bone fragility

Authors: ${ }^{\mathrm{A}}$ academic clinical fellow, MRC Lifecourse Epidemiology Unit, University of Southampton, Southampton, UK; ${ }^{\mathrm{C}}$ clinical research fellow, MRC Lifecourse Epidemiology Unit, University of Southampton, Southampton, UK, and Paediatric Endocrinology, Southampton University Hospitals NHS Foundation Trust, Southampton, UK; ' professor of musculoskeletal epidemiology, MRC Lifecourse Epidemiology Unit, University of Southampton, Southampton, UK; ${ }^{\text {D }}$ rofessor of rheumatology and clinical epidemiology, MRC Lifecourse Epidemiology Unit, University of Southampton, Southampton, UK, and NIHR Southampton Biomedical Research Centre, University of Southampton and University Hospital Southampton NHS Foundation Trust,

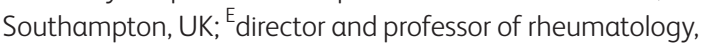
MRC Lifecourse Epidemiology Unit, University of Southampton, Southampton, UK, NIHR Southampton Biomedical Research Centre, University of Southampton and University Hospital Southampton NHS Foundation Trust, Southampton, UK, and NIHR Musculoskeletal Biomedical Research Unit, University of Oxford, Oxford, UK and propensity to fracture. Worldwide, there are nearly 9 million osteoporotic fractures each year, and the US Surgeon General's report of 2004, consistent with data from the UK, suggested that almost one in two women and one in five men will experience a fracture in their remaining lifetime from the age of 50 years. ${ }^{1}$ The cost of osteoporotic fracture in the UK approaches $\mathfrak{£} 3$ billion annually and, across the EU, the estimated total economic cost of the approximately 3.5 million fragility fractures in 2010 was $€ 37$ billion. $^{2}$ In this article we review the pathogenesis of osteoporosis and approaches to improving bone strength, aimed at reducing the immense burden of osteoporotic fracture.

\section{Pathogenesis}

\section{Hierarchical structure}

Fractures occur when the force applied to a bone exceeds its strength. A bone needs to be both stiff and flexible to resist fracture, which is achieved through a hierarchical structure. Collagen type-1 fibrils are wound in a triple helical structure, linked together with non-collagenous proteins, which help to prevent shearing. Hydroxyapatite crystals deposited on the collagen structure add strength, particularly in compression. Cross-linkage between collagen fibrils with non-collagenous proteins is reduced in osteoporotic bone, leading to reduced tensile strength. ${ }^{3}$ In addition, larger hydroxyapatite crystals are found in osteoporosis, making bone more brittle and prone to fracture. ${ }^{4}$

\section{Bone cells}

Osteoblasts, osteocytes and osteoclasts are the three main types of bone cells. Osteoblasts are bone forming and may become embedded within bone mineral as mature osteocytes (comprising 90-95\% of the cells within bone) or remain on the surface as bone-lining cells. Osteoclasts are multinucleated cells responsible for bone resorption. Osteoblasts and osteoclasts work together in a coordinated fashion at specific sites on the surface of trabecular or cortical bone, forming 'bone multicellular units'. During bone formation, osteoblasts lay down new osteoid collagen matrix and over a period of weeks to months, crystals of calcium hydroxyapatite form on the collagen fibrils. Bone is laid down during growth and repair and through adaptation to mechanical loading in a process known as modelling. 
Remodelling, in contrast, involves a cycle of resorption and formation of existing bone. Osteocytes play a key role in the regulation of modelling and remodelling. The arrangement of the osteocytes around Haversian canals acts as a mechanosensory system and allows communication both directly between neighbouring osteocytes and through the release of endocrine, paracrine and autocrine signalling factors to other bone cells. The various pathways important to the regulation of osteoblast and osteoclast activity, such as receptor activator of nuclear factor kappa B-receptor activator of nuclear factor kappa B ligand (RANK-RANKL) and Wnt signalling, are increasingly recognised as targets for anti-osteoporosis agents.

\section{Changes in bone structure across the life course}

The balance of formation and resorption has a critical influence on bone mass and strength throughout life. There is a positive balance during childhood until achievement of peak bone mass in early adulthood, ${ }^{5}$ with a subsequent period of stability and then a negative balance in older age, with osteoclast activity greater than osteoblast activity, leading to bone loss. In women, this process is accelerated after the menopause. At the level of the whole bone, the cellular mechanisms and associated influences result in differences in structure between males and females, and alterations with advancing age. Males typically have a larger bone cross-sectional area than females, and in addition there is a significant reduction in cortical thickness in females following the menopause, contributing to the well established sex differences in fracture risk. The structure of the trabeculae differs between the sexes, with young women having fewer and thinner trabeculae than young men, and a greater reduction in trabecular number in women as they age. ${ }^{6}$ In addition, cortical porosity increases at a faster rate in female ageing. ${ }^{7}$

\section{Table 1. Risk factors for osteoporosis.}

\begin{tabular}{|c|c|}
\hline $\begin{array}{l}\text { Risk factors independent } \\
\text { of BMD }\end{array}$ & $\begin{array}{l}\text { Risk factors dependent } \\
\text { on BMD }\end{array}$ \\
\hline Age & $\begin{array}{l}\text { Untreated hypogonadism, } \\
\text { premature menopause }\end{array}$ \\
\hline $\begin{array}{l}\text { Previous personal history of } \\
\text { fragility fracture }\end{array}$ & Malabsorption \\
\hline $\begin{array}{l}\text { Maternal history of hip fracture } \\
\text { - heritable influences }\end{array}$ & $\begin{array}{l}\text { Endocrine disease eg } \\
\text { hyperthyroidism }\end{array}$ \\
\hline Glucocorticoid therapy & Chronic renal disease \\
\hline Smoking & Chronic liver disease \\
\hline Alcohol intake $\geq 3$ units/day & $\begin{array}{l}\text { Chronic obstructive pulmonary } \\
\text { disease }\end{array}$ \\
\hline Rheumatoid arthritis & Immobility \\
\hline Body mass index $\leq 19 \mathrm{~kg} / \mathrm{m}^{2}$ & $\begin{array}{l}\text { Drugs eg androgen deprivation } \\
\text { therapy, aromatase inhibitors }\end{array}$ \\
\hline \multicolumn{2}{|l|}{ Falls } \\
\hline \multicolumn{2}{|l|}{ Caucasian } \\
\hline $\begin{array}{l}\text { Geography - latitudes furthest } \\
\text { from equator }\end{array}$ & \\
\hline
\end{tabular}

Table 2. The spectrum of antifracture efficacy of
pharmacological interventions for osteoporosis.

\begin{tabular}{llll} 
Intervention & Vertebral & Non-vertebral & Hip \\
Alendronate & + & + & + \\
Risedronate & + & + & + \\
Zoledronic acid & + & + & + \\
Etidronate & + & - & - \\
Ibandronate & + & $+^{*}$ & - \\
Raloxifene & + & - & - \\
Strontium ranelate & + & + & $+^{*}$ \\
Teriparatide & + & + & - \\
Denosumab & + & + & + \\
\hline "post hoc analysis in a subset of patients. & &
\end{tabular}

\section{Clinical risk factors}

There are many factors that influence fracture risk, either through bone mineral density (BMD) or through independent mechanisms. These include age, glucocorticoid therapy, a previous personal history of fracture, a family history of hip fracture, current smoking practice, alcohol abuse and certain diseases associated with osteoporosis eg rheumatoid arthritis, diabetes, osteogenesis imperfecta in adults, untreated longstanding hyperthyroidism, hypogonadism or premature menopause ( $<45$ years), chronic malnutrition, malabsorption and chronic liver disease. These are summarised in Table 1 , and have, in terms of risk assessment, been incorporated into the FRAX ${ }^{\circledR}$ tool, ${ }^{8}$ a WHO supported initiative which uses risk factors, with or without BMD measurement, to estimate a 10-year probability of either hip fracture or major osteoporotic fracture. FRAX ${ }^{\circledR}$ is by far the most commonly used such tool globally, covering $75 \%$ of the world's population, and may, as in the UK, be linked to assessment algorithms to define thresholds for intervention with treatment. ${ }^{9}$

\section{Treatment of osteoporosis}

\section{Vitamin D and calcium supplementation}

The role of vitamin D and calcium supplementation has been much debated in recent years with numerous meta-analyses suggesting conflicting findings. A large UK randomised controlled trial demonstrated that supplementation with either calcium, vitamin D or both for secondary fracture prevention at the population level appeared ineffective, ${ }^{10}$ although supplementation in high-risk settings where deficiencies are expected, for example in nursing homes, may be beneficial. ${ }^{11}$ A recent individual patient data meta-analysis demonstrated that overall, there appeared to be a modest benefit for combined vitamin D and calcium supplementation for hip fractures, total fractures and probably vertebral fractures, but that there was no benefit for vitamin D alone. ${ }^{12}$ Although there has been discussion from one research group that excess calcium intake may be associated with increased cardiovascular risk, ${ }^{13}$ this has not been substantiated across many other studies. Indeed, it is reassuring to note that a recent individual-patient-data 
meta-analysis of the anti-fracture studies suggests that calcium and vitamin D supplementation in combination is associated with an improvement in mortality, which is not observed with vitamin D supplementation alone. ${ }^{14}$ Almost all of the randomised control trial evidence for the efficacy of antiosteoporosis drugs comes from patients who were prescribed concomitant calcium and vitamin D supplementation; both should therefore usually be prescribed adjunctively with treatment for osteoporosis.

\section{Bisphosphonates}

Bisphosphonates are synthetic analogues of the naturally occurring compound pyrophosphate and bind strongly to hydroxyapatite, inhibiting bone resorption by inactivating osteoclasts. The most commonly prescribed oral bisphosphonate is alendronate. If taken properly (in the morning with a glass of water, 45 minutes before food, drink or other medications and remaining upright for 30-60 minutes after the dose), upper GI side effects are uncommon. However, for those who are unable to tolerate oral bisphosphonates, or in whom they are contraindicated (for example malabsorption or dysphagia), then an intravenous bisphosphonate, such as zoledronate (given yearly in a dose of $5 \mathrm{mg}$ by infusion over a minimum of 15 minutes) is an alternative.

\section{Denosumab}

Denosumab, a fully humanised antibody to RANKL, is a newer antiresorptive agent. RANKL, secreted by osteoblasts, is a major activator of osteoclastic bone resorption and mimics the action of osteoprotegerin. Denosumab is administered as a subcutaneous injection once every six months and its efficacy has been demonstrated in patients with renal impairment, although due consideration should be given to the possibility of underlying renal bone disease in chronic kidney disease $4-5$. Three-year fracture data show a $68 \%$ reduction in vertebral fracture and $40 \%$ reduction in hip fracture. ${ }^{15}$ Side effects are uncommon, and may rarely include skin infections, predominantly cellulitis. This is not typically seen at the injection site and may be secondary to an immunomodulatory effect of the drug. Hypocalcaemia can also be a risk, particularly if the patient is vitamin $\mathrm{D}$ deficient, or has renal impairment.

\section{Strontium ranelate}

Strontium, an element directly below calcium in group 2 of the periodic table, is combined with ranelic acid as a carrier to form strontium ranelate. It is taken as a single daily oral dose. Its mechanism of action remains a subject of research, but there is evidence that it increases bone strength by altering bone material properties. Administration of strontium ranelate leads to a substantial increase in BMD at the spine and hip, though part of this increase is artefactual, due to incorporation of strontium (which has a greater atomic weight than calcium) into bone. Studies have shown a 36\% relative risk reduction in hip fracture over three years in osteoporotic patients. ${ }^{16}$ In 2013 a Medicines and Healthcare Products Regulatory Agency warning on strontium ranelate was issued due to increased risk of cardiovascular disorders (relative risk for myocardial infarction: 1.6), in addition to the previously known risk of venous thromboembolism (VTE). Therefore its use is now restricted to treatment of severe osteoporosis in postmenopausal women with high risk of fracture and in men at increased risk of fracture, but with no cardiovascular or cerebrovascular disease. However, within this selected group of patients, particularly now that many individuals have undergone long-term bisphosphonate treatment, strontium ranelate does still offer a useful alternative. ${ }^{17}$

\section{Selective oestrogen receptor modulators - raloxifene}

Raloxifene is a selective oestrogen receptor modulator that has antiresorptive oestrogenic effects on the skeleton without the unwanted risks of oestrogen in the breast. Its use is also associated with a significant decrease in the risk of breast cancer. It has been shown to be effective in preventing postmenopausal bone loss and in preventing vertebral fractures. However there is no evidence that raloxifene prevents hip or non-vertebral fractures. ${ }^{18}$ Adverse effects include leg oedema, cramps, hot flushes and a two- to three-fold increase in the risk of VTE.

\section{Teriparatide}

Teriparatide (recombinant human 1-34 parathyroid-hormone peptide) is the only agent in current widespread use with truly anabolic effects on bone. It is administered by subcutaneous injection in daily doses of $20 \mu \mathrm{g}$. It increases bone formation and produces large increases in BMD, leading to approximately $70 \%$ reduction in the incidence of new moderate or severe vertebral fractures over 18 months of treatment, together with reductions in non-vertebral fractures. ${ }^{19}$ Side effects are uncommon but may include nausea, headache and dizziness; in addition, transient hypercalcaemia and hypercalciuria may occur. Studies have shown added benefit in combination treatments such as teriparatide plus denosumab or teriparatide plus zoledronate, ${ }^{20}$ although such approaches are not yet approved clinically in the UK, and use of teriparatide in the UK is currently limited to those older patients at highest fracture risk and who may have failed other therapies.

\section{Adverse effects and duration of therapy}

Atypical femoral factures of the subtrochanteric region and femoral shaft may also rarely occur in patients taking bisphosphonates or denosumab. These are usually located in the lateral cortex around which endosteal thickening may be observed prior to fracture occurrence. Individuals may have prodromal pain and fractures typically are transverse, sometimes bilateral and occur after minimal trauma. Although these fractures can occur in bisphosphonate-/denosumab-naïve individuals, they appear more commonly in patients taking these therapies for a prolonged duration. It is thought that the reason for this increased incidence is related to oversuppression of bone turnover. Overall, the fractures prevented greatly outnumber those atypical events potentially resulting from medication. ${ }^{21}$ Osteonecrosis of the jaw is extremely rarely observed during therapy for osteoporosis (as low as $1 / 100,000$ / year for individuals on oral bisphosphonates), ${ }^{22}$ but appears more commonly when higher doses of bisphosphonates are 
given intravenously for treatment of bone metastases. A causal link to bisphosphonates is unproven but international guidance suggests a prudent approach, encouraging patients to maintain good oral hygiene and have regular dental visits, with invasive dental work performed before commencement of bisphosphonate or denosumab therapy. ${ }^{23}$

Current UK guidance has therefore moved towards a reassessment of the need for treatment after three years of intravenous bisphosphonate/subcutaneous denosumab, and five years of oral bisphosphonate. ${ }^{9}$ For high-risk patients, continuation of treatment is usually warranted, but where there have been no incident fractures and BMD has improved, a period without treatment may be recommended.

\section{Novel therapies}

\section{Cathepsin-K inhibitors}

Odanacatib is a once weekly oral treatment for osteoporosis in which phase-III clinical trials have recently been performed. It inhibits cathepsin-K, a cysteine protease expressed in osteoclasts which degrades type-1 collagen. In postmenopausal women with low BMD, 24 months of treatment with odanacatib was shown to produce increases in lumbar spine and total-hip BMD by $5.5 \%$ and $3.2 \%$, respectively, whereas BMD at these sites was essentially unchanged with placebo $(-0.2 \%$ and $-0.9 \%) .{ }^{24}$ However, later trial outcomes also demonstrated a possible increase in cerebrovascular events, which has led to a delay in FDA approval while outcomes are further adjudicated.

\section{Anti-sclerostin antibodies}

Sclerostin, an osteocyte-secreted protein, negatively regulates osteoblasts and inhibits bone formation through the lowdensity lipoprotein receptor-related protein 5 (LRP5)/ Wnt signalling pathway. The role of sclerostin in bone mass homeostasis was highlighted by the finding that two rare high-bone-mass diseases, van Buchem disease and sclerostosis have been linked to inactivating mutations in the sclerostin gene. A monoclonal antibody against sclerostin, romosozumab, was administered intravenously to healthy men and postmenopausal women and was shown to increase boneformation markers, along with a dose-related decrease in boneresorption markers. Statistically significant increases in BMD of up to $5.3 \%$ at the lumbar spine and $2.8 \%$ at the total hip compared with placebo were observed on day 85 of treatment. ${ }^{25}$

A randomised, double-blind, placebo-controlled multicentre phase-II clinical trial of a second agent, blosozumab (a humanised monoclonal antibody targeted against sclerostin), demonstrated statistically significant dose-related increases in spine, femoral neck and total hip BMD as compared with placebo. In the highest dose group, BMD increases from baseline reached $17.7 \%$ at the spine, and $6.2 \%$ at the total hip. Biochemical markers of bone formation also increased during blosozumab treatment, while resorption markers decreased. ${ }^{26}$

These findings present promise for future use of anti-sclerostin antibodies as anabolic osteoporosis therapies. Other potential areas for investigation include new selective oestrogen receptor or androgen receptor modulators, calcilytics (calcium receptor antagonists), the nitric oxide pathway and interventions aimed at sarcopenia, in what is an exciting and rapidly changing field of osteoporosis treatment and fracture prevention.

\section{Conclusion}

In recent decades, our understanding of the pathogenesis of osteoporosis has dramatically increased, with development of a wide range of effective pharmaceutical approaches to fracture prevention. Given the enormous prevalence of osteoporosis, and frequency and burden of resulting fragility fractures, one of the key concerns going forward remains the optimal identification of those requiring treatment. ${ }^{27}$ The tools for fracture risk assessment are widely available, in the form of the FRAX ${ }^{\circledR}$ calculator, and new medications are undergoing testing. It is vital that awareness of osteoporosis is promoted among all health professionals, in order to ensure closure of the so-called 'treatment gap', and with a consequent reduction in the burden of osteoporotic fracture for individuals, healthcare systems and societies.

\section{Acknowledgements}

We would like to thank Medical Research Council (UK), Arthritis Research UK, National Osteoporosis Society (UK), International Osteoporosis Foundation and NIHR for supporting this work.

\section{Note}

This article was originally published in the 2015 Clinical Medicine supplement Horizons in Medicine 27. All articles in this supplement are available at www.clinmed.rcpjournal.org/content/15/Suppl_6

\section{References}

1 van Staa TP, Dennison EM, Leufkens HG, Cooper C. Epidemiology of fractures in England and Wales. Bone 2001;29:517-22.

2 Hernlund E, Svedbom A, Ivergard M et al. Osteoporosis in the European Union: medical management, epidemiology and economic burden : A report prepared in collaboration with the International Osteoporosis Foundation (IOF) and the European Federation of Pharmaceutical Industry Associations (EFPIA). Arch Osteoporos 2013;8:136.

3 Saito M, Marumo K. Collagen cross-links as a determinant of bone quality: a possible explanation for bone fragility in aging, osteoporosis, and diabetes mellitus. Osteoporos Int 2010;21:195-214.

4 Boskey A. Bone mineral crystal size. Osteoporos Int 2003;14 Suppl 5:S16-20; discussion S-1.

5 Hernandez CJ, Beaupre GS, Carter DR. A theoretical analysis of the relative influences of peak BMD, age-related bone loss and menopause on the development of osteoporosis. Osteoporos Int 2003;14:843-7.

6 Macdonald HM, Nishiyama KK, Kang J et al. Age-related patterns of trabecular and cortical bone loss differ between sexes and skeletal sites: a population-based HR-pQCT study. J Bone Miner Res 2011;26:50-62.

7 Zebaze RM, Ghasem-Zadeh A, Bohte A et al. Intracortical remodelling and porosity in the distal radius and post-mortem femurs of women: a cross-sectional study. Lancet 2010;375:1729-36.

8 Kanis JA, Johnell O, Oden A, Johansson H, McCloskey E. FRAX and the assessment of fracture probability in men and women from the UK. Osteoporos Int 2008;19:385-97.

9 Compston J, Bowring C, Cooper A et al. Diagnosis and management of osteoporosis in postmenopausal women and older men in the UK: National Osteoporosis Guideline Group (NOGG) update 2013. Maturitas 2013;75:392-6.

10 Grant AM, Avenell A, Campbell MK et al. Oral vitamin D3 and calcium for secondary prevention of low-trauma fractures in elderly people (Randomised Evaluation of Calcium Or vitamin D, RECORD): a randomised placebo-controlled trial. Lancet 2005;365:1621-8. 
11 Chapuy MC, Pamphile R, Paris E et al. Combined calcium and vitamin D3 supplementation in elderly women: confirmation of reversal of secondary hyperparathyroidism and hip fracture risk: the Decalyos II study. Osteoporos Int 2002;13:257-64.

12 DIPART (Vitamin D Individual Patient Analysis of Randomized Trials) Group. Patient level pooled analysis of 68500 patients from seven major vitamin D fracture trials in US and Europe. BMJ 2010;340:b5463.

13 Bolland MJ, Avenell A, Baron JA et al. Effect of calcium supplements on risk of myocardial infarction and cardiovascular events: meta-analysis. BMJ 2010;341:c3691.

14 Rejnmark L, Avenell A, Masud T et al. Vitamin D with calcium reduces mortality: patient level pooled analysis of 70528 patients from eight major vitamin D trials. J Clin Endocrinol Metab 2012;97:2670-81.

15 Cummings SR, Martin JS, McClung MR et al. Denosumab for Prevention of Fractures in Postmenopausal Women with Osteoporosis. N Engl J Med 2009;361:756-65.

16 Reginster JY, Seeman Vernejoul MCD E et al. Strontium ranelate reduces the risk of nonvertebral fractures in postmenopausal women with osteoporosis: treatment of peripheral osteoporosis (TROPOS) study. J Clin Endocrinol Metab 2005;90:2816-22.

17 Reginster JY, Brandi ML, Cannata-Andia J et al. The position of strontium ranelate in today's management of osteoporosis. Osteoporos Int 2015;26:1667-71.

18 Ettinger B, Black DM, Mitlak BH et al. Reduction of vertebral fracture risk in postmenopausal women with osteoporosis treated with raloxifene: results from a 3-year randomized clinical trial. JAMA 1999;282:637-45.

19 Neer RM, Arnaud CD, Zanchetta JR et al. Effect of parathyroid hormone (1-34) on fractures and bone mineral density in postmenopausal women with osteoporosis. N Engl J Med 2001;344:1434-41.
20 Tsai JN, Uihlein AV, Lee $\mathrm{H}$ et al. Teriparatide and denosumab, alone or combined, in women with postmenopausal osteoporosis: the DATA study randomised trial. Lancet 2013;382:50-6.

21 Shane E, Burr D, Abrahamsen B et al. Atypical subtrochanteric and diaphyseal femoral fractures: second report of a task force of the American Society for Bone and Mineral Research. J Bone Miner Res 2014;29:1-23.

22 Khan AA, Morrison A, Hanley DA et al. Diagnosis and management of osteonecrosis of the jaw: a systematic review and international consensus. J Bone Miner Res 2015;30:3-23.

23 Khosla S, Burr D, Cauley J et al. Bisphosphonate-associated osteonecrosis of the jaw: report of a task force of the American Society for Bone and Mineral Research. J Bone Miner Res 2007;22:1479-91.

24 Bone HG, McClung MR, Roux C et al. Odanacatib, a cathepsin-K inhibitor for osteoporosis: A two-year study in postmenopausal women with low bone density. J Bone Miner Res 2010;25:937-47.

25 Padhi D, Jang G, Stouch B et al. Single-dose, placebo-controlled, randomized study of AMG 785, a sclerostin monoclonal antibody. J Bone Miner Res 2011;26:19-26.

26 Recker RR, Benson CT, Matsumoto T et al. A randomized, doubleblind phase 2 clinical trial of blosozumab, a sclerostin antibody, in postmenopausal women with low bone mineral density. J Bone Miner Res 2015;30:216-24.

27 Kanis JA, Svedbom A, Harvey N, McCloskey EV. The osteoporosis treatment gap. J Bone Miner Res 2014;29:1926-8.

Address for correspondence: Professor C Cooper, MRC Lifecourse Epidemiology Unit, University of Southampton, Southampton General Hospital, Southampton S016 6YD, UK. Email:cc@mrc.soton.ac.uk
'Smoking is the biggest avoidable cause of death and disability, and social inequality in health, in the UK.'

\section{Nicotine without smoke Tobacco harm reduction}

Since e-cigarettes became available in the UK in 2007, their use has been surrounded by medical and public controversy. This new 200-page report from the Royal College of Physicians examines the science, public policy, regulation and ethics surrounding e-cigarettes and other nontobacco sources of nicotine, and addresses these controversies and misunderstandings with conclusions based on the latest available evidence.

ISBN 978-1-86016-600-6 £15 including $p+p$ or free to download

\section{Royal College} of Physicians

\section{Download the report: rcplondon.ac.uk/nicotine}

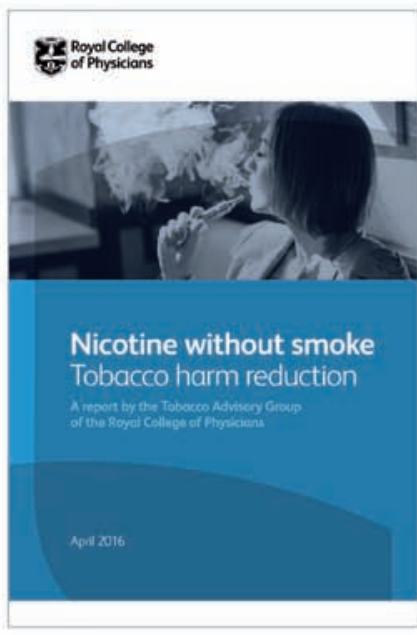

Order a copy: shop.rcplondon.ac.uk 